\title{
Tramas tróficas de peces de arrecifes en la región noroccidental de Cuba. II. Grupos funcionales
}

\author{
Ivet Hernández, Consuelo Aguilar \& Gaspar González Sansón \\ Centro de Investigaciones Marinas, Universidad de La Habana, Calle 16. e/ 1ra y 3ra. No. 114, Miramar, Ciudad de \\ La Habana, Cuba; ycabrera@cim.uh.cu
}

Recibido 12-VI-2007. Corregido 30-VI-2008. Aceptado 31-VII-2008.

\begin{abstract}
Trophic webs of reef fishes at Cuba's NW area. II. Functional groups. A conceptual model of the food webs, mainly with fish, was built in a rocky shore of Havana City with data covering from October 2004 to February 2006. The stomach contents of the most abundant fish was complemented with the literature. We used the Relative Importance Index method to describe diets of carnivorous and omnivorous fish; and a modification of the Relative Abundance method for sponge eaters and herbivorous fish. Agglomerative numeric classification techniques were used to determine the diet's similarity. The matrix was made using Bray-Curtis dissimilarity index. Ten functional trophic groups were formed on the basis of diet similarity. The cascade effect is evidenced in this area by the big top predator shortage and the dominance of a few low trophic level species. The regulator role of top predators is low because of over-fishing: the trophic web of this area is highly altered. Rev. Biol. Trop. 56 (3): 1391-1401. Epub 2008 September 30.
\end{abstract}

Key words: reef fish, trophic webs, cascade effect, functional trophic groups, Cuba.

Se ha señalado como un efecto de la sobrepesca a nivel mundial, la rápida disminución de las poblaciones de peces depredadores (Bellwood et al. 2003, Myers y Worm 2003) y una disminución del nivel trófico de los organismos capturados, lo que determina una modificación de la estructura comunitaria (Pauly et al. 1998). Esto habita debido a que la estructura de las comunidades de un ecosistema natural está sometido a las dos clases de regulaciones descritas por Scheffer et al. (2005): el control desde arriba (depredación) y el control desde abajo (disponibilidad de alimento). Ambos controles influyen sobre las poblaciones y regulan su abundancia y composición. Si el control desde abajo es el limitante (disponibilidad de alimento insuficiente), priman las especies oportunistas. Por su parte, la depredación también es un importante mecanismo regulador, ya que puede distorsionar los patrones de asentamiento y limitar la abundancia (Ringler 1993, Steele y Forrester 2002). En un trabajo realizado por Jennings y Polunin (1997) sobre los efectos de la pesca en la abundancia y diversidad de los peces de arrecife, encontraron que los piscívoros mayores de $30 \mathrm{~cm}$ disminuyeron su abundancia de manera importante con el aumento de la pesca. Concluyeron que la depredación de estas especies dejó de ser importante en la estructuración de las comunidades de peces del arrecife. Los piscívoros tienen un rol regulatorio muy importante en los sistemas marinos, por lo tanto, la sobrepesca de estos puede tener ramificaciones sobre la comunidad restante (Hixon y Carr 1997). La disminución del primer control por ausencia de depredadores debido a la sobrepesca, produce que sólo el control desde abajo pase a jugar un papel determinante y ocasione una reestructuración de la comunidad. Esto puede observarse de manera directa como cambios en la alimentación y provoca 
variaciones en las cadenas tróficas y los flujos de energía (Friedlander y DeMartini 2002). En Cuba se han realizado diversos estudios acerca de la alimentación en peces (Sierra y Díaz-Zaballa 1984, Pastor 2000, Faloh 2001, Aguilar 2005), pero no existen trabajos que integren las tramas alimentarias como un todo en las zonas arrecifales. En el litoral rocoso de Ciudad de La Habana, las comunidades de peces han sido modificadas notablemente producto de la sobrepesca, lo cual se evidencia por la ausencia, casi total, de depredadores de talla grande como las especies pertenecientes a las familias Lutjanidae, Serranidae y Sphyraenidae (Aguilar 2005). El presente trabajo elabora un modelo de las tramas alimentarias de esa zona con énfasis en la ictiofauna.

\section{MATERIALES Y MÉTODOS}

El área de estudio es la porción del sublitoral de Ciudad de La Habana $\left(23^{\circ} 7.587^{\prime} \mathrm{N}\right.$ $82^{\circ} 25.793^{\prime} \mathrm{W}$ ), entre los 2 y $18 \mathrm{~m}$ de profundidad. Los muestreos se realizaron diariamente entre octubre del 2004 y febrero del 2006. Los peces fueron capturados mediante buceo autónomo empleando jamos de malla plástica tejida con una trama de $1 \mathrm{~mm}^{2}$ para los individuos pequeños y arpón para los ejemplares de talla mediana y grande. Los especimenes se transportaron al laboratorio, donde se les determinó el peso húmedo en gramos con balanzas digitales tipo Denver de $1 \mathrm{mg}$ de precisión y la longitud de horquilla en $\mathrm{cm}$ con un ictiómetro. Posteriormente se realizó la extracción de los estómagos, los cuales fueron conservados en solución de formalina al $10 \%$ hasta su análisis.

Cada entidad alimentaria encontrada se clasificó por un grupo de expertos, hasta el taxon más bajo posible empleando un microscopio estereoscópico. Los ítems alimentarios del contenido estomacal fueron secados con papel de filtro, contadas, medidas y pesadas. Para describir las dietas de las especies carnívoras y omnívoras (consumen materia animal y vegetal), se empleó el método del Índice de Importancia Relativa (IRI), definido como IRI
$=(\% \mathrm{~N}+\% \mathrm{~W}) \mathrm{X} \% \mathrm{~F}$ por Pinkas et al. (1971) $\mathrm{y}$ el porcentaje de IRI como \%IRI $=100 \mathrm{x}$ $\operatorname{IRI}_{\mathrm{i}} / \Sigma^{\mathrm{n}}{ }_{\mathrm{i}=1}$ IRI $_{\mathrm{i}}$ (Morato et al. 2003). Para los herbívoros y comedores de esponjas se usó una modificación del método de abundancia relativa (Suárez et al. 1989). Se empleó una escala del uno al tres con el objetivo de disminuir los sesgos definida como: 1- Escaso, 2- Normal, 3- Abundante. Para determinar el grado de similitud en la dieta de los peces, se emplearon técnicas de análisis de clasificación numérica jerárquica aglomerativa,. La formación de los grupos tróficos afines se basó en el método de ligamiento promedio. La matriz fue construida empleando el índice de disimilitud de BrayCurtis. Ningún dato fue transformado ni estandarizado. Los análisis se realizaron usando el programa PRIMER 5.2.8 para Windows.

\section{RESULTADOS}

Fueron analizados 1.367 estómagos, de los cuales el $5 \%$ estaba vacío. Se analizaron 22 especies de peces clasificadas como "carnívoras y omnívoras" y diez especies "herbívoras y comedoras de esponjas". Estos peces fueron los más abundantes, de más fácil captura y poco crípticos (Cuadro 1). Las tallas medias de todos los peces oscilaron entre los 4 y $40 \mathrm{~cm}$ de longitud total. Estas longitudes se consideran pequeñas y evidencian la ausencia de especies de tallas grandes (mayores que $50 \mathrm{~cm}$ ), lo cual concuerda con lo planteado por Aguilar (2005).

\section{Análisis de la similitud de las dietas. Carnívoros y omnívoros}

De acuerdo con el análisis de disimilitud por el método IRI, se obtuvieron siete grupos, los cuales presentan un porcentaje de superior al $60 \%$ (Fig. 1). Estos son caracterizados por:

Grupo 1: se caracteriza por que sus integrantes son predominantemente ictiófagos (\%IRI=32.94), especialmente Cephalopholis fulva, Rypticus saponaceus, Lutjanus apodus, L. synagris, Myripristis jacobus, Caranx ruber 
TABLA 1

Especies seleccionadas para la investigación

TABLE 1

Species chosen for research

Especies

Acanthostracion polygonius (Poey, 1876)

Acanthurus bahianus (Walbaum, 1792)

Acanthurus chirurgus (Bloch, 1787)

Acanthurus coeruleus (Bloch y Schneider,1801)

Bothus lunatus (Linnaeus, 1758)

Cantherhines pullus (Ranzani, 1842)

Caranx ruber (Bloch, 1793)

Cephalopholis fulva (Linnaeus, 1758)

Chaetodon capistratus (Linnaeus, 1758)

Chaetodon ocellatus (Bloch, 1787)

Chaetodon striatus (Linnaeus, 1758)

Chromis multilineata (Guichenot, 1853)

Diodon holocanthus (Linnaeus, 1758)

Equetus acuminatus (Bloch y Schneider,1801)

Haemulon plumieri (Lacepéde, 1801)

Haemulon sciurus (Shaw, 1803)

Halichoeres garnoti (Valenciennes, 1839)

Holacanthus ciliaris (Linnaeus, 1758)

Holocentrus adscensionis (Osbeck, 1765)

Neoniphon marianus (Cuvier, 1829)

Holocentrus rufus (Walbaum, 1792)

Lutjanus apodus (Walbaum, 1792)

Lutjanus synagris (Linnaeus, 1758)

Myripristis jacobus (Cuvier, 1829)

Pomacanthus paru (Bloch, 1787)

Rypticus saponaceus (Bloch y Schneider,1801)

Sparisoma aurofrenatum (Valenciennes, 1840)

Sparisoma chrysopterum (Bloch y Schneider,1801)

Sparisoma viride (Bonnaterre, 1788)

Serranus tigrinus (Bloch, 1790)

Stegastes partitus (Poey, 1868)

Thalassoma bifasciatum (Bloch, 1791)

\begin{tabular}{|c|c|}
\hline Nombre vulgar & Código \\
\hline Torito hexagonal & Lacpol \\
\hline Barbero & Acabah \\
\hline Barbero & Acachi \\
\hline Barbero azul & Acacoe \\
\hline Lenguado & Botlun \\
\hline Lija & Canpul \\
\hline Civí carbonero & Carrub \\
\hline Guatívere & Cepful \\
\hline Parche ocelado & Chacap \\
\hline Parche amarillo & Chaoce \\
\hline Parche rayado & Chastr \\
\hline Cromis prieto & Chrmul \\
\hline Pez erizo & Diohol \\
\hline Vaqueta rayada & Equacu \\
\hline Ronco arará & Haeplu \\
\hline Ronco amarillo & Haesci \\
\hline Doncella de cara amarilla & Halgar \\
\hline Angelote reina & Holcil \\
\hline Carajuelo de ascensión & Holasd \\
\hline Carajuelo mariano & Neomar \\
\hline Carajuelo rufo & Holruf \\
\hline Cají & Lutapo \\
\hline Biajaiba & Lutsyn \\
\hline Candil barreado & Myrjac \\
\hline Angelote francés & Pompar \\
\hline Jaboncillo & Rypsap \\
\hline Loro & Spaaur \\
\hline Loro & Spachr \\
\hline Loro & Spavir \\
\hline Serrano tigre & Sertig \\
\hline Chopita bicolor & Stepar \\
\hline Doncella cara de cotorra & Thabif \\
\hline
\end{tabular}

La clasificación se realizó siguiendo los criterios de Eschmeyer (1998), para los nombres científicos y de Guitar (1978), para los nombres comunes.

Scientific names classification was according to Eschmeyer (1998). Vulgar names classification was according to Guitar (1978). 


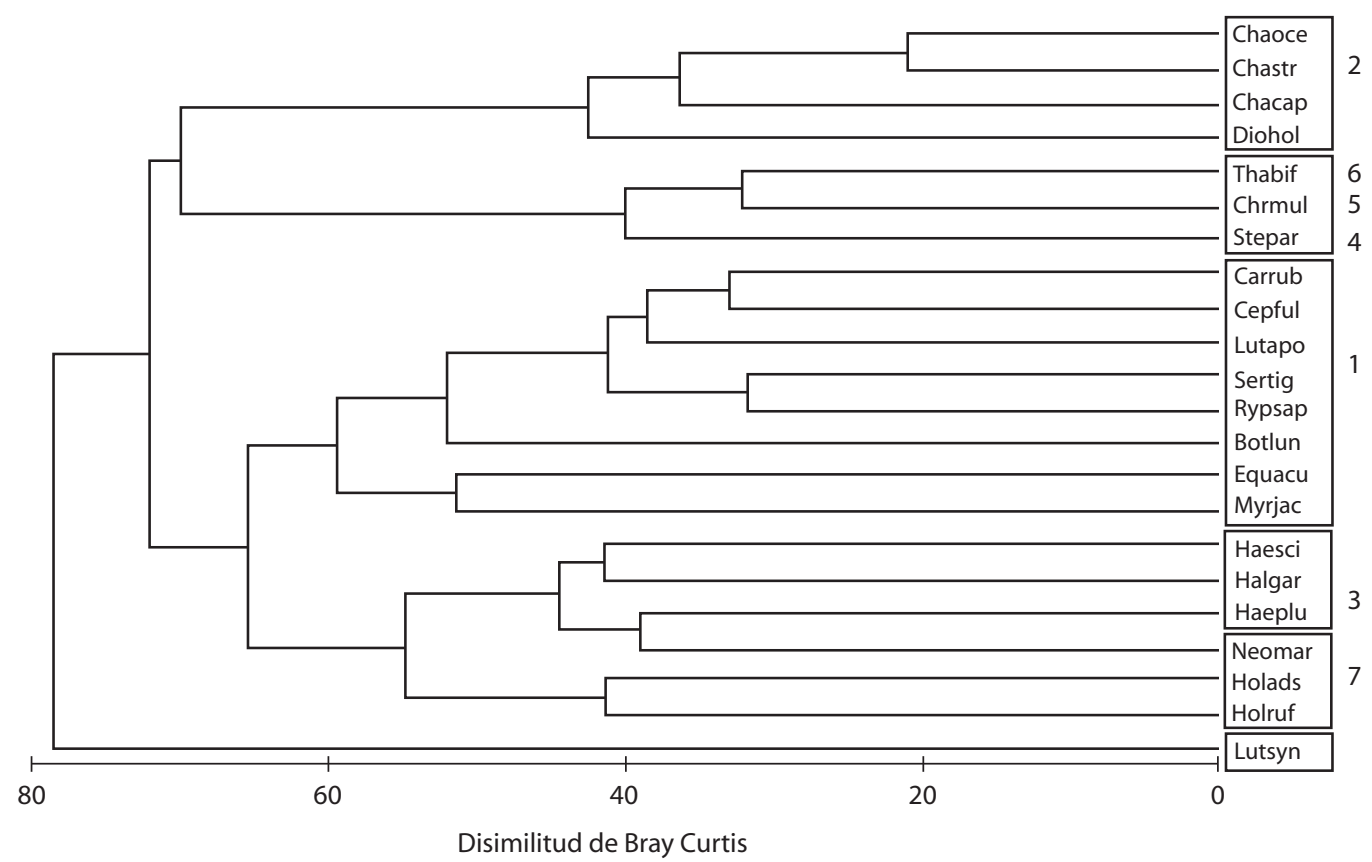

Fig. 1. Dendrograma con la clasificación de las especies carnívoras; basado en la composición de sus dietas expresada por el método IRI. Los números expresan los grupos formados.

Fig. 1. Carnivorous species classification based on diet composition according to Importance Relative Index. Numbers express the resulting groups.

y Bothus lunatus. Consumen como entidades comunes, peces como Acanthurus spp, Aulostomus maculatus, Canthigaster rostrata y Scarus spp, entre otros. La mayoría de los peces encontrados en el contenido estomacal de estos peces resultaron ser los estadios juveniles de las especies presa (menos de $5 \mathrm{~cm}$ ). Las especies de este grupo consumen, además, camarones $(\% \mathrm{IRI}=17.06)$, principalmente del género Periclimenes (\%IRI=5.2), Los braquiuros, generalmente xántidos $(\% \mathrm{IRI}=18.56)$, son ingeridos fundamentalmente por Bothus lunatus, Serranus tigrinus y Equetus acuminatus. Este grupo de especies se denominarán "consumidores de peces y crustáceos grandes”.

Grupo 2: otra agrupación es la formada por las especies del género Chaetodon. Estos peces comen crinoideos, que se supone que sean juveniles debido a que su disco central mide menos de $1 \mathrm{~cm}$ de diámetro $(\% \mathrm{IRI}=20.9)$. También consumen poliquetos (\%IRI=26.8), especialmente capitélidos por Chaetodon striatus. Los peces del género Chaetodon conforman el grupo de los "consumidores de invertebrados pequeños".

Grupo 3: este se forma por los roncos (Haemulidae) y Halichoeres garnoti (Labridae), los cuales se alimentan fundamentalmente de braquiuros, especialmente májidos $(\% \mathrm{IRI}=28.8)$ y moluscos gastrópodos (\%IRI=20.9), fundamentalmente Halichoeres garnoti. También consumen otros crustáceos (\%IRI=5.2), fundamentalmente Haemulon plumieri. El grupo de los roncos y $H$. garnoti queda definido como "consumidores de moluscos y decápodos". La especie Diodon holacanthus se caracterizó por alimentarse de moluscos gastrópodos $(\% \mathrm{IRI}=4.25)$; algas $(\% \mathrm{IRI}=13.37)$, especialmente 
Ulva; huevos demersales, braquiuros, poliquetos, erizos, poríferos, entre otros. Esta especie queda incluida en este grupo.

Grupo 4: Thalassoma bifasciatum, Chromis multilineata y Stegastes partitus aparecen agrupados por el IRI, pero en esta agrupación se deben evaluar ciertas características. Estas especies se caracterizan por ingerir huevos demersales (\%IRI=13.57), fundamentalmente $S$. partitus; huevos pelágicos (\%IRI=11.33), generalmente C. multilineata y copépodos calanoides $(\% \mathrm{IRI}=21.7)$, especialmente $T$. bifasciatum. Es menester considerar que la mayoría de estas entidades son de origen planctónico y no fueron identificadas hasta taxa inferiores, por lo que se asume que en estos casos se pierde la capacidad discriminatoria del método de agrupación empleado. $S$. partitus, aunque presenta la misma dieta de crustáceos muy pequeños, camarones, poliquetos y copépodos calanoides que T. bifasciatum, incluye también algas, especialmente del género Polysiphonia y abundantes cianofitas, por lo que se define como "omnívoro" y se le incluye en un grupo separado.

Grupo 5: Por otra parte, Chromis multilineata ingiere foraminíferos y copépodos calanoides, ciclopoides y harparticoides planctónicos. Teniendo en cuenta este criterio, se define a C. multilineata como "planctófago", formando un grupo aparte.

Grupo 6: A partir de las consideraciones anteriores, es lógico considerar que Thalassoma bifasciatum forme un grupo diferente como "comedor de crustáceos pequeños".

Grupo 7: Para las especies del género Holocentrus se obtienen resultados dispersos. Consumen fundamentalmente braquiuros, generalmente májidos, exceptuando a Neoniphon marianus que se alimenta de braquiuros más pequeños y en menor cuantía y consume algas. Todos estos resultados concuerdan con los encontrados anteriormente para la especie. Teniendo en cuenta esta información se definen a las especies del género Holocentrus y Neoniphon como "consumidores de crustáceos medianos".

\section{Análisis de la similitud de las dietas. Herbívoros y consumidores de esponjas}

Los datos obtenidos mediante el método de abundancia relativa permitieron agrupar a los herbívoros en tres grupos (Fig. 2). Todas las especies herbívoras consumen Halimeda $(\% \mathrm{AR}=6.77)$. No se encontró una clasificación acertada para definir subgrupos dentro de la clasificación de "herbívoros".

Grupo 8: Las especies del género Acanthurus se caracterizan por alimentarse de una gran cantidad de algas rojas (Agardhiella, Centroceras, Ceramium, Galaxaura, Gelidiella, Gelidiopsis, Heterosiphonia), especialmente Jania $(\% \mathrm{AR}=7.58)$. Además consumen algas pardas como Dictyota $(\% \mathrm{AR}=6.05)$, cianofitas $(\% \mathrm{AR}=6.69)$ y otros $(\% \mathrm{AR}=18.14)$, particularmente $A$. chirurgus y A. coerelus. Para estos casos la categoría "otros" está compuesta en un $37.52 \%$ por arena. Estas especies poseen estómagos de paredes muy fuertes. Incluyen grandes cantidades de materia inorgánica en su dieta como piedras pequeñas y arena, las cuales son ingeridas tal vez, para ayudar en la maceración del alimento vegetal (Randall 1967). Los integrantes de esta familia consumen además, determinados grupos de algas, como las del género Polysiphonia y Enteromorpha, que no son las más abundantes de la zona. Teniendo en cuenta esta información, puede considerarse a los barberos (Acanthurus spp), como herbívoros selectivos. Estos resultados coinciden totalmente con los encontrados por Horn (1989).

Grupo 9: Las especies del género Sparisoma consumen más Centroceras $(\% \mathrm{AR}=6.01)$, Galaxaura $(\% \mathrm{AR}=9.5)$, materia orgánica $(\% \mathrm{AR}=16.3)$ y otros $(\% \mathrm{AR}=14.6)$. La dieta de este grupo es similar a la del grupo anterior en cuanto a las especies de algas que ingieren pero estas son consumidas en magnitudes y combinaciones muy diferentes, lo que 


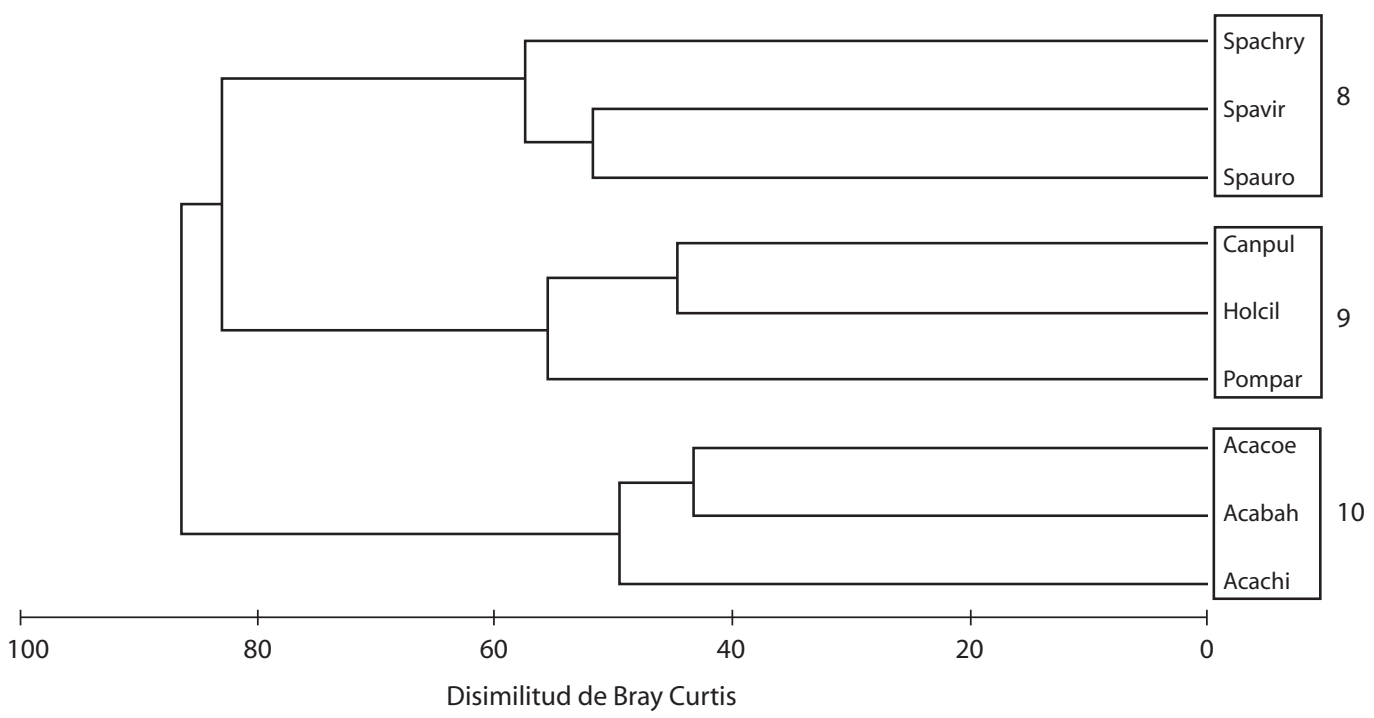

Fig. 2. Dendrograma que muestra los resultados de la clasificación de las especies herbívoras y comedoras de esponjas, basado en la composición de sus dietas expresada por el método de abundancia relativa. Los números expresan los grupos formados.

Fig. 2. Herbivorous and sponge eaters' species classification results based on diets composition according to Relative Abundance method. Numbers express the resulting groups.

les permite segregar los subnichos tróficos y disminuir la competencia. En esta investigación, se encontró que el $3 \%$ del contenido estomacal de los peces está formado por entidades que son consumidas de forma accidental. Diversos autores (Hiatt y Strasburg 1960, Horn 1989), plantean que los integrantes de la familia Scaridae son capaces de ingerir las algas que crecen sobre los corales y les provee de una ventaja sobre el resto de los herbívoros en condiciones de escasez de algas. Teniendo esto en cuenta, no es extraño que el $2 \%$ del contenido estomacal analizado en este estudio, estuviese conformado por arena. Ocasionalmente, animales pequeños como crustáceos, moluscos y poliquetos fueron encontrados en los estómagos de los herbívoros, concordando con Horn (1989) y Sierra et al. (2001). Considerando el tamaño de estas entidades, resulta evidente que no fueron seleccionadas por el pez, sino que fueron ingeridas por azar.

Hiatt y Strasburg (1960), reportaron que todos los especímenes de la familia Scaridae se caracterizaban por raspar los pólipos vivos de los corales. En esta investigación no se encontraron cantidades significantes de pólipos de coral en ningún estómago de especies de esta familia. Los pocos pólipos hallados, pudieron ser ingeridos accidentalmente, lo cual es consistente con lo planteado por diferentes autores (Randall 1967, Horn 1989). Los integrantes de la familia Scaridae han sido considerados herbívoros selectivos (Horn 1989).

Grupo 10: Las especies Holacanthus ciliaris, Cantherhines pullus y Pomacanthus paru son principalmente comedoras de esponjas (\%AR=37.87), particularmente Holacanthus ciliaris. Dichas esponjas conforman el $58.5 \%$ de su dieta. Randall (1967) y Aburto-Oropeza et al. (2000), reportaron que la mayoría de las esponjas consumidas por los representantes de la familia Pomacanthidae presentan gran cantidad de espículas silíceas. Se podría esperar que dichas espículas dañaran el estómago de los peces pero esto puede que sea evitado, tal vez, 
por las importantes cantidades de mucus que secretan estos animales en sus tractos digestivos. Todos estos datos concuerdan con los obtenidos durante el presente estudio. Además consumen Halimeda, Caulerpa, Dictyopteris, Dictyota, Gelidiopsis, entre otras. En la dieta de Cantherhines pullus pueden encontrarse además, algunos restos de erizos, hidrozoos y materia orgánica.

\section{MODELO CONCEPTUAL DE LA TRAMA ALIMENTARIA}

Con la información obtenida se elaboró un esquema (Fig. 3) de las relaciones tróficas fundamentales que involucran a la ictiofauna de la zona de estudio. Basado en el IRI se determinó la importancia de las entidades dentro de la dieta general de los peces. Las flechas indican el flujo de energía. Cephalopholis fulva y Caranx ruber, principalmente, actúan como los depredadores tope en esta comunidad. Los herbívoros se encuentran resaltados dentro del cuadro de líneas discontinuas y ocupan el nivel trófico más bajo.

Opitz (1996), elaboró una trama trófica general para un arrecife poco impactado por el hombre, señalando que la ictiofauna de los arrecifes se caracteriza por tener 3 grupos tróficos principales: 1 , depredadores tope de gran tamaño y nado rápido, con hábitat predominantemente pelágico y migraciones extensas; 2, los carnívoros dominantes y 3 , los herbívoros dominantes. Esta trama es muy similar a la obtenida en este trabajo, excepto por el último nivel trófico, el cual estaría ocupado por especies pequeñas (máximo $30 \mathrm{~cm}$ ) y que, en su mayoría, no son estrictamente ictiófagas. Debido a lo anterior, resulta lógico encontrar sólo peces pequeños en el contenido estomacal de la mayoría de los ictiófagos. Teniendo esta información en cuenta, se consideraron como grupos separados los adultos y los jóvenes de las especies de las familias Scaridae y Acanthuridae. La dieta de los jóvenes no fue analizada en esta investigación. Los adultos de estas familias no son consumidos de manera importante por ninguna especie de la zona y sólo los ejemplares jóvenes (menos de $10 \mathrm{~cm}$ ) se consideran presas de los depredadores tope. También es posible que, teniendo en cuenta los cambios ontogénicos de la dieta de los peces (Hoines y Bergstad 1999, Pastor 2000, Sierra et al. 2001, Baldo y Drake 2002), los jóvenes puedan no encontrarse en los mismos grupos tróficos que los adultos. Las especies Caranx ruber, Myripristis jacobus y Bothus lunatus presentaron peces adultos en sus estómagos pero estos tampoco eran de gran tamaño (como en el caso de Pseudupeneus maculatus y Canthigaster rostrata, que medían menos de $15 \mathrm{~cm}$ ). Los pocos adultos que fueron consumidos en su talla máxima, corresponden a especies pequeñas (Stegastes partitus y Halichoeres spp).

Está bien documentado que los depredadores tope pueden considerarse reguladores de las poblaciones de peces (Webster 2002, Santucci y Wahl 2003, Beukers-Stewart y Jones 2004, Scheffer et al. 2005). Sin embargo, en la zona de estudio, Thalassoma bifasciatum y Stegastes partitus conforman el $63 \%$ de los peces de la zona (Hernández-Hernández et al. 2006), y apenas son consumidos. Estos resultados sugieren que estas especies presentan ventajas adaptativas y que al tener poca regulación por parte de los depredadores, dominan ampliamente en la comunidad.

Los únicos depredadores encontrados para Stegastes partitus, fueron ejemplares grandes $(50 \mathrm{~cm})$ de la especie Caranx ruber. Esta es una especie secundaria debido a su carácter transitorio en el arrecife y a la baja abundancia de los individuos mayores. Esto provoca que su impacto regulador sobre la ictiofauna sea muy limitado. No se encontraron depredadores para la especie Thalassoma bifasciatum.

En general, en el área de estudio, incluso los mayores depredadores se alimentan de crustáceos. Esto indica que todo un nivel trófico está ausente debido, tal vez a la sobrepesca a la que está sometida toda el área. Las especies depredadoras tope son pocas y presentan una abundancia baja (Aguilar 2005). Esto trae como consecuencia que el control desde arriba en esta zona sea deficiente o nulo, permitiendo que otras especies de niveles tróficos inferiores 


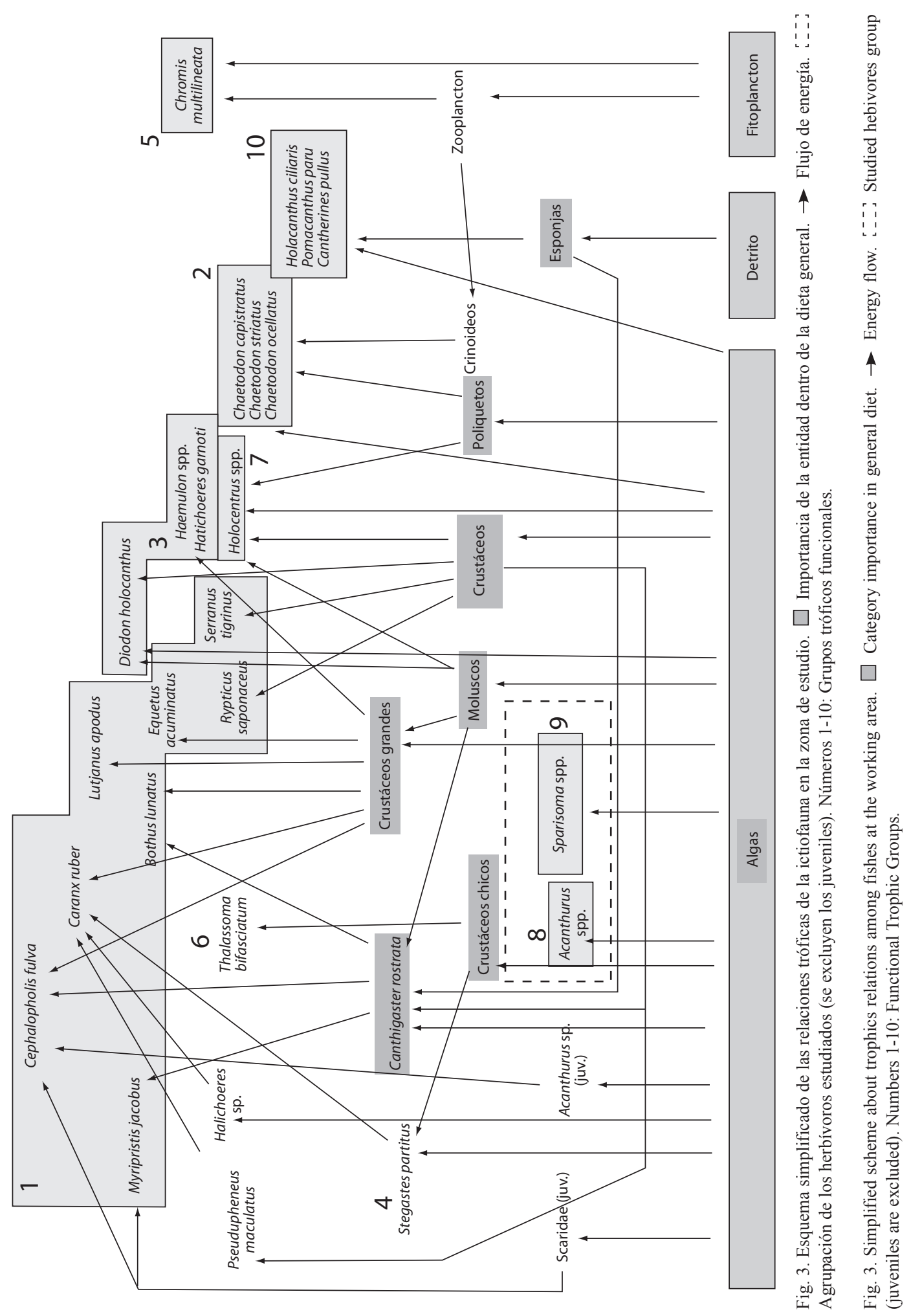


aumenten su abundancia. Diferentes autores (Jackson et al. 2001, Friedlander y DeMartini 2002, Gardner et al. 2003), han reportado que este incremento de la abundancia en los peces de más bajo nivel trófico, puede ocasionar que se varíen incluso las bases alimentarias del ecosistema. Los mismos autores definen este fenómeno como el "efecto cascada". Los resultados en la presente investigación evidencian la presencia de este efecto en el área de estudio.

Opitz (1996) expresó que los individuos de un nivel trófico pueden ser sustituidos fácilmente por otros que tengan características energéticas similares, o sea, que su biomasa y producción sean semejantes, netamente, a las de las especies que sustituyen. Así, especies más pequeñas pero abundantes pueden ocupar el sitio de depredadores mayores cuando estos son eliminados del ecosistema. Estas especies de bajo nivel trófico se tornan más abundantes e incluso pueden llegar a ser dominantes en una determinada región.

A respecto, no se conoce información sobre cómo se integrarían las tramas tróficas de la ictiofauna dominante de un arrecife afectado por sobrepesca. La poca literatura existente sobre cadenas alimentarias y flujo de energía (Opitz 1996, Sierra et al. 2001), son realizadas en zonas menos impactadas y la mayoría emplean criterios de agrupación para los peces que resultan demasiado amplios. Grupos funcionales como herbívoros, carnívoros o bentófagos resultan totalmente acertados, pero teniendo en cuenta que el $80 \%$ de los peces del arrecife son bentófagos, esta agrupación puede resultar a veces poco discriminatoria. Vega-Cendejas et al. (1994), propuso la formación de cinco grupos funcionales para un conjunto de 12 especies de dieta homogénea, capturadas con redes en una playa. Estos grupos fueron: los comedores de microcrustáceos, comedores de poliquetos, piscívoros, omnívoros y herbívoros.

La formación de diez grupos tróficos funcionales brinda la posibilidad de que los mismos sean más específicos y explicativos. Los calificativos de "bentófagos" ó "comedores de crustáceos grandes", no transmiten la misma información. El segundo término indica que el individuo tratado, no sólo es bentófago, sino también identifica mejor el grupo de entidades consumidas y da una idea de la posible fisonomía del pez, como sus dimensiones y el tamaño de la boca, entre otros. La formación de menos cantidad de grupos implica ampliar los calificativos para que estos abarquen todas las entidades consumidas por los peces, pero debido a que generalmente los peces presentan dietas muy heterogéneas, los términos que las describen, se tornan vagos. Por otra parte, manejar más de diez grupos puede resultar engorroso, puesto que se superponen. Los grupos tróficos funcionales no siguen completamente la distribución sistemática. Las especies pertenecientes a las familias Chaetodontidae, Scaridae y Acanthuridae coinciden con esta distribución. Las restantes familias, al tener dietas más heterogéneas, son clasificadas siguiendo un criterio de selección diferente al empleado en la sistemática.

\section{RESUMEN}

Se elaboró un modelo conceptual de la trama alimentaria en una zona del sublitoral rocoso de Ciudad de la Habana, con énfasis en los peces desde octubre del 2004 hasta febrero del 2006. Para ello se emplearon los contenidos estomacales de las especies más abundantes y la información disponible en la literatura. Para describir las dietas de las especies de peces carnívoras y omnívoras se empleó el índice de importancia relativa. Para los herbívoros y consumidores de esponjas se usó una modificación del método de abundancia relativa. Se emplearon técnicas de análisis de clasificación numérica jerárquica aglomerativa para determinar el grado de similitud en las dietas de los peces. La matriz fue construida empleando el índice de disimilitud de Bray-Curtis. El análisis de la similitud de las dietas permitió la formación de 10 grupos tróficos funcionales. La escasez de depredadores tope de gran tamaño y la dominancia de muy pocas especies de bajo nivel trófico, evidencian el efecto cascada en la zona estudiada. La trama trófica del área se encuentra muy alterada, debido a que el papel regulador de la ictiofagia está muy disminuido.

Palabras clave: peces de arrecife, cadenas tróficas, efecto cascada, grupos tróficos funcionales, Cuba.

\section{REFERENCIAS}

Aburto-Oropeza, O., E. Sala \& C. Sanchez-Ortiz. 2000. Feeding behaviour, habitat use, and abundance of 
the angelfish Holacanthus passer (Pomacanthidae) in the southern Sea of Cortes. Environ. Biol. Fish. 57: 435-442.

Aguilar, C. 2005. La ictiofauna costera de Ciudad de La Habana: Efectos acumulativos de agentes estresantes múltiples en varios niveles de organización biológica. Tesis de Doctorado, Centro de Investigaciones Marinas, Universidad de La Habana, Cuba.

Baldo, F. \& P. Drake. 2002. A multivariate approach to the feeding habits of small fishes in the Guadalquivir Estuary. J. Fish Biol. 61: 21-32.

Bellwood, D.R., A.S. Hoey \& J.H. Choat. 2003. Limited functional redundancy in high diversity systems: resilience and ecosystem function on coral reefs. Ecol. Lett. 6: 281-285.

Beukers-Stewart, B.D. \& G.P. Jones. 2004. The influence of prey abundance on the feeding ecology of two piscivorous species of coral reef fish. J. Exp. Mar. Biol. Ecol. 299: 155-184.

Eschmeyer, W.N. 1998. Catalog of Fishes. Spec. Pub. No.1 Centre Biod. Res. and California Acad. Sci. III. T. 2905 pp.

Faloh, I.G. 2001. Alimentación natural de Stegastes partitus (Poey, 1868), en el sublitoral de Ciudad de La Habana. Trabajo de Diploma. Centro de Investigaciones Marinas, Universidad de La Habana, Cuba.

Friedlander, A.M. \& E.E. DeMartini. 2002. Contrasts in density, size, and biomass of reef fishes between the northwestern and the main Hawaiian islands: the effects of fishing down apex predators. Mar. Ecol. Prog. Ser. 230: 253-264.

Gardner, T.A., I.M. Cote, J.A. Gill, A. Grant \& A.R. Watkinson. 2003. Long-term region-wide declines in Caribbean corals. Science 301: 958-960.

Guitart, J.D. 1978. Sinopsis de los peces marinos de Cuba. Cuba, Científ-Técnica, La havana. 881 pp.

Hernández-Hernández, I., C. Aguilar \& G. GonzálezSansón. 2006. Variación estacional de la abundancia de especies de peces seleccionadas en el sublitoral rocoso de Ciudad de La Habana, Cuba. Rev. Invest. Mar. 27: 61-68.

Hiatt, R.W. \& D.W. Strasburg. 1960. Ecological relationships of the fish fauna on coral reefs of the Marshall Islands. Ecol. Monogr. 30: 65-127.

Hixon, M.A. \& M.H. Carr. 1997. Synergistic predation, density dependence, and population regulation in marine fish. Science 277(5328): 946-949.
Hoines A.S. \& O.A. Bergstad. 1999. Resource sharing among cod, haddock, saithe and pollack on a herring spawning ground. J. Fish Biol., 55: 1233-1257.

Horn, M.H. 1989. Biology of marine herbivorous fishes. Oceanogr. Mar. Biol. Annu. Rev. 27: 167-272.

Jackson, J.B.C., M.X. Kirby, W.H. Berger, K.A. Bjorndal, L.W. Botsford, B.J. Bourque, R.H. Bradbury, R. Cooke, J. Erlandson, J.A. Estes, T.P. Hughes, S. Kidwell, C.B. Lange, H. S. Lenihan, J.M. Pandoli, C.H. Peterson, R.S. Steneck, J. Tegner \& R.R. Warner. 2001. Historical overfishing and the recent collapse of coastal ecosystems. Science 293: 629638.

Jennings, S. \& N.V.C. Polunin. 1997. Impact of predator depletion by fishing on the biomass and diversity of non-target reef fish communities. Coral Reef 16 : 71-82.

Morato, T., E. Solá, M.P. Grós \& G. Menezes. 2003. Diets of thornback ray (Raja clavata) and tope shark (Galeorhinus galeus) in the bottom longline fishery of the Azores, northeastern Atlantic. Fish. Bull. 101: 590-602.

Myers, R.A \& B. Worm. 2003. Rapid worldwide depletion of predatory fish communities. Nature 423: 280-283.

Opitz, S. 1996. Trophic interactions in Caribbean Coral Reefs. ICLARM Tech. Rep. 43.

Pastor G., L. 2000. Características biológicas de cinco especies de peces marinos ornamentales. Tesis de Maestría, Centro de Investigaciones Marinas, Universidad de La Habana, Cuba.

Pauly, D., V. Christensen, J. Dalsgaard, R. Froese \& F. Torres, Jr. 1998. Fishing down marine food webs. Science 279: 860-863.

Pinkas, L., M.S. Oliphant \& J.L.K. Iverson. 1971. Food habits of albacore, bluefin tuna and bonito in California waters. California. Fish and Game Fish. Bull. 152: 1-105.

Randall, J.E. 1967. Food habits of reef fishes of the West Indies. Stud. Trop. Oceanogr. 5: 665-847.

Ringler, N.H. 1993. Trophic Relationships of Fishes and Other Aquatic Organisms. Nordiske-Seminar-ogArbejdsrapporter 572: 30-52.

Santucci, V.J. \& D.H. Wahl. 2003. The Effects of Growth, Predation, and First-Winter Mortality on Recruitment of Bluegill Cohorts. Trans. Am. Fish. Soc. 132: 346360. 
Scheffer M., S. Carpenter \& B. Young. 2005. Cascading effects of overfishing marine systems. Ecol. Evol., 20: $579-581$.

Sierra, L.M. \& J. Díaz-Zaballa. 1984. Alimentación de 2 especies de sardina Harengula humeralis (Cuvier, 1829) y Harengula clupeola (Cuvier, 1829), en la costa norte de la Ciudad de La Habana (rada del Instituto de Oceanología). Rep. Invest. Oceanol. Acad. Cienc. Cuba 3: 1-19.

Sierra, L.M., R. Claro \& O.A. Popova. 2001. Trophic biology of the marine fishes of Cuba, p. 115-148. In R. Claro, K.C. Lindeman \& L.R. Parenti (eds.). Ecology of the marine fishes of Cuba. Smithso. Instit., Washington and London, England.
Steele, M.A. \& G.E. Forrester. 2002. Early postsettlement predation on three reef fishes: Effects on spatial patterns of recruitment. Ecol. 83: 1076-1091.

Suárez, A.M, C. Aguilar \& G. González-Sansón. 1989. Comparación de dos métodos para la cuantificación del fitobentos. Rev. Invest. Mar. 10: 21-26.

Vega-Cendejas, M.E., M. Hernández \& F. ArreguinSanchez. 1994. Trophic interrelations in a beach seine fishery from the northwestern coast of the Yucatan peninsula, Mexico. J. Fish Ecol. 44: 647-659.

Webster, M.S. 2002. Factors affecting the dynamics and regulation of coral- reef fish populations (Gramma loreto). Diss. Abst. Int. Pt. B. Sci. Eng. 62: 4338. 
